Cahiers $d u$ MONDE RUSSE

\section{Cahiers du monde russe}

Russie - Empire russe - Union soviétique et États indépendants

$46 / 4 \mid 2005$

L'invention d'une politique humanitaire

\title{
Balázs Apor, Jan C. Behrends, Polly Jones, E. A. Rees, eds., The Leader Cult in Communist Dictatorships | Klaus Heller, Jan Plamper, eds., Personality Cults in Stalinism
}

\section{Gábor T. Rittersporn}

\section{(2) OpenEdition}

\section{Journals}

Édition électronique

URL : https://journals.openedition.org/monderusse/6632

DOI : $10.4000 /$ monderusse. 6632

ISSN : $1777-5388$

Éditeur

Éditions de l'EHESS

\section{Édition imprimée}

Date de publication : 1 décembre 2005

Pagination : 931-935

ISBN : 2-7132-2057-2

ISSN : $1252-6576$

Référence électronique

Gábor T. Rittersporn, « Balázs Apor, Jan C. Behrends, Polly Jones, E. A. Rees, eds., The Leader Cult in Communist Dictatorships | Klaus Heller, Jan Plamper, eds., Personality Cults in Stalinism », Cahiers du monde russe [En ligne], 46/4 | 2005, mis en ligne le 29 juin 2009, consulté le 03 septembre 2022. URL : http://journals.openedition.org/monderusse/6632; DOI : https://doi.org/10.4000/monderusse.6632

Ce document a été généré automatiquement le 3 septembre 2022.

Tous droits réservés 


\title{
Balázs Apor, Jan C. Behrends, Polly Jones, E. A. Rees, eds., The Leader Cult in Communist Dictatorships | Klaus Heller, Jan Plamper, eds., Personality Cults in Stalinism
}

\author{
Gábor T. Rittersporn
}

\section{RÉFÉRENCE}

Balázs APOR, Jan C. BEHRENDS, Polly JONES, E. A. REES, eds., The Leader Cult in

Communist Dictatorships. Stalin and the Eastern Bloc. Basingstoke, New York :

Palgrave Macmillan, 2004, $298 \mathrm{p}$.

Klaus HELLER, Jan PLAMPER, eds., Personality Cults in Stalinism/Personenkulte im Stalinismus. Göttingen : V\&R Unipress, 2004, 472 p.

1 Nous sommes habitués aux images de foules enfiévrées saluant le leader d'un État communiste. Néanmoins, un passage du journal intime de Kornej Čukovskij a de quoi laisser rêveur. L'écrivain n'avait rien d'un inconditionnel du bolchevisme ; pourtant, en 1936, il décrit la joie extatique qui s'empara de lui à la vue de Stalin. Čukovskij ne se croyait pas capable d'un tel débordement d'émotion. Son ami Boris Pasternak, également présent, partageait ses sentiments et lui chuchotait des paroles exaltées. À en croire le journal, les deux écrivains jubilaient...

On a peu étudié la dévotion vouée aux dirigeants communistes. Les livres recensés ici sont issus de colloques dont la thématique centrale portait sur le culte des leaders communistes.

3 Balázs Apor et ses collègues brossent un panorama du culte des dirigeants suprêmes en Allemagne de l'Est, Pologne, Hongrie, dans les Balkans et bien entendu en Union 
soviétique. En Europe de l'Est, la glorification des meilleurs élèves de Stalin n'a pas toujours suivi le modèle soviétique et l'effet n'était pas nécessairement comparable à celui obtenu dans le grand pays frère.

Isabella Main, Marcin Zaremba et Jan C. Behrends expliquent qu'il n'était pas facile de susciter l'enthousiasme des masses en Pologne où le parti a néanmoins persévéré, jusqu'en 1980, dans sa velléité de rendre populaires les premiers secrétaires. Behrends montre qu'il fallait éviter que la mise en scène de la dévotion à Stalin en RDA ne ressemblât trop au culte de Hitler, mais le résultat laissa à désirer. Árpád von Klimó et Balázs Apor soulignent que la confection de l'image du père intrépide, modeste et bienveillant des Hongrois resta artisanale. Dans les pays de l'Est, les machines de la propagande avaient manqué de temps pour être bien rodées et la période du haut stalinisme fut trop brève pour que le culte envahisse le quotidien des populations.

Tel ne fut pas le cas en URSS. E. A. Rees prend l'exemple soviétique pour montrer que les privations que la population eut à endurer ne furent pas propices à l'élaboration d'un consensus autour du régime ; mais que, malgré tout, l'État-parti réussit à rassembler les masses en utilisant une figure fédératrice. Selon l'auteur, les traditions prérévolutionnaires jouèrent un certain rôle dans la réception du message, mais selon une formulation savamment modernisée.

6 Benno Ennker, dans sa contribution au livre d'Apor et al. qu'il poursuit dans le deuxième ouvrage, décrit un processus complexe où l'entourage immédiat de Stalin est à l'origine de la vénération institutionnalisée du guide. Ce sont ses lieutenants qui l'élaborent, de manière plutôt empirique, à la fois parce qu'ils reconnaissent le pouvoir du chef et qu'ils sont en compétition permanente pour gagner ses faveurs.

7 Les travaux de Malte Rolf, également présents dans les deux recueils, changent de perspective. Rolf explore le culte des dirigeants locaux et l'espace où se déploient les rites autour de leurs personnes. Dans le modèle qu'il élabore, la célébration des émules de Stalin fonctionne comme une stratégie de communication: il s'agit de signaler que le pouvoir des chefs locaux n'a pas moins d'importance que celui de la capitale.

8 L'étude de Catriona Kelly porte sur la diffusion de l'image de Stalin comme garant d'une enfance heureuse. Si ce portrait ne manquait pas de fasciner les plus jeunes, ses dimensions devenaient écrasantes et il risquait de s'édulcorer avec le temps. Le Stalin de Sarah Davies comprend bien les avantages du culte qui lui est voué, autant que le risque qu'il ne tourne au ridicule; si bien qu'il s'efforce d'apparaitre comme un politicien modeste, conscient toutefois de son importance, et de répéter, même à ses collaborateurs les plus proches, que l'histoire n'est pas l'œuvre de personnalités éminentes. Le Stalin de Jan Plamper transcende les barrières nationales de l'URSS multiethnique. Sa gloire est chantée dans toutes les langues du pays. Mais si son habit, avant la guerre, rappelle la tenue de simple soldat, après la victoire, il porte l'uniforme du généralissime et apparaît comme dirigeant supranational, père de tous les peuples.

9 L'ouvrage que Plamper a édité avec Klaus Heller est ambitieux. Les éditeurs s'aventurent au-delà de la sphère soviétique et donnent la parole à des auteurs qui repensent l'expérience de l'Italie fasciste et de l'Allemagne nazie. De même, les contributeurs ne se limitent pas au culte de Stalin en tant que tel. Ils explorent l'arrière-fond historique ainsi que les représentations de Stalin dans les pièces de théâtre et dans les films. Quittant les sentiers battus, ils vont jusqu'à chercher les traces du dictateur dans des jeux de rôle que la propagande porte aux nues. 
10 L'introduction de Plamper est dans l'esprit de ce vaste projet. L'objectif est de rechercher les racines de la vénération moderne des hommes illustres dans le dévouement romantique à l'individu génial et de remonter à la généalogie du terme «culte de la personnalité » jusqu'au début du $\mathrm{xx}^{\mathrm{e}}$ siècle. Il s'agit aussi de revisiter les interprétations traditionnelles $\mathrm{du}$ charisme des grands chefs: hypothèses des constantes anthropologiques, de la religion politique et du recours utilitaire à une autorité absolue. L'auteur exprime ses réserves quant à la validité de ces approches. Il souligne le caractère syncrétique du culte de Stalin, qu'on a quelque mal à réduire aux schémas habituels.

Plamper consacre un chapitre au culte est-allemand du leader soviétique, qui témoigne du caractère multidimensionnel du phénomène. Ses origines remontent à l'attente d'un homme providentiel aux temps wilhelmiens, puis à l'époque de Weimar et même jusqu'à la République fédérale. Le mouvement ouvrier allemand avait par tradition le culte de ses héros et de ses dirigeants, de sorte que les communistes purent se passer du modèle soviétique. Le conditionnement de la population à l'enthousiasme pour Hitler n'était pas sans importance, quoiqu'il pût, appliqué au commandant suprême des vainqueurs, susciter l'hostilité. Les réactions ambiguës à des événements comme la mort de Stalin montrent que rien ne serait plus simpliste que de penser l'attitude des masses en termes dichotomiques.

12 Simonetta Falasca-Zamponi explique qu'il est difficile de comprendre le culte de Mussolini sans prendre en compte les techniques cinématographiques utilisées pour créer son image. Elle relève la conjonction du grand spectacle, du politique et de la culture de masse qui métamorphosait les citoyens en consommateurs d'un divertissement populaire. Henning Bühmann met également l'accent sur les aspects médiatiques de la présentation de Hitler comme créateur d'une Allemagne nouvelle. Les propagandistes nazis se sont inspirés de l'année liturgique chrétienne en instituant un cycle de fêtes qui commémoraient l'histoire du parti national-socialiste et qui culminaient en une parade gigantesque présidée par le Führer. Il s'agissait d'établir un lien personnel entre les masses et Hitler, lien qui ne pouvait fonctionner que jusqu'aux défaites militaires du régime. Bühmann rappelle que le concept weberien du pouvoir charismatique s'accommode mal avec le cas nazi. Selon Weber, l'autorité charismatique procède de qualités personnelles authentiques et n'a pas besoin de médiatisation. Les historiens de l'URSS pourraient méditer ce raisonnement.

Barbara Walker se penche sur un précédent notable du culte soviétique du leader. Les cercles et les réseaux personnels des élites professionnelles et intellectuelles de l'époque prérévolutionnaire sont inimaginables sans les célébrités qui les guidaient et qui étaient révérées en tant que mentors. Ce n'est pas un hasard si Stalin a acquis l'épithète de " maître ». Tout autant que l'intelligentsia de l'Ancien Régime, les bolcheviks éprouvaient le besoin d'être rassemblés autour d'un guide. Irina Paperno estime qu'un intellectuel sophistiqué comme Čukovskij n'était pas forcément un adepte docile. Mais il suffisait qu'il accepte le système soviétique comme une nécessité historique pour que Stalin lui apparaisse comme la personnification d'un processus irrésistible qui n'est pas sans rappeler l'œuvre divine. Incarnation d'un projet grandiose, la figure de Stalin finissait par posséder une force d'attraction quasi charnelle qui attachait l'individu au pouvoir. Paperno interprète les textes dans lesquels les intellectuels en venaient à dénoncer Stalin comme l'expression d'une volonté de rompre un lien émotionnel en se détachant du vécu soviétique. 

canonique de Stalin prenne vie sur scène. En revanche, le cinéma était le medium rêvé pour ranimer l'icône. Nikolas Hüllbusch fait revivre le leader cinématographique ainsi que les scénarios et les images habilement agencés pour populariser ses vertus. Dans l'imagerie des Soviétiques, le Stalin du cinéma remplaçait le dirigeant inaccessible.

Lubov' Orlova, l'actrice culte du cinéma stalinien, correspondait à cet idéal. L'analyse d'Oksana Bulgakova ne s'arrête pas à l'héroïne des films, dans lesquels une modeste travailleuse accède aux sommets de la gloire grâce à ses efforts et à la générosité du pouvoir soviétique. L'auteur va au-delà pour faire ressortir la subtilité des techniques publicitaires mises en œuvre et le personnage qu'elles ont créé. On a produit une comédienne fort attirante dont la séduction toutefois ne devait pas dépasser les limites étroites que le régime avait tracées à tout ce qui ressemblait à de l'érotisme.

Il importait par ailleurs que l'exemple de la plus illustre vedette de l'URSS soit consacré par la tradition historique. Ce n'est pas un hasard si c'est au cinéma qu'a incombé la tâche de suggérer que le patron du Kremlin était l'héritier légitime des lauriers d'un souverain mémorable. Frithjof Benjamin Schenk suit le destin d'Aleksandr Nevskij au pays des Soviets: après avoir été honni dans un premier temps comme autocrate chauvin, oppresseur de la nation et saint d'une Église que les bolcheviks haïssaient, il a fini par être célébré à la veille de la guerre comme homme d'État clairvoyant, patriote ardent, père généreux du peuple et défenseur valeureux de la Rus'. Schenk décrit également les péripéties du scénario de Sergej Ejzenštejn et Petr Pavlenko, qui durent adapter leur récit au cours politique du régime. À l'origine, le film sur Aleksandr Nevskij devait se terminer par la mort du héros. En haut lieu, on fit néanmoins savoir qu'un bon prince ne devait pas mourir.

17 Une question demeure, qui mérite réflexion. On peut se demander dans quelle mesure le terme de " culte de la personnalité » suffit à rendre compte de la problématique des deux ouvrages. Jan Plamper note que l'usage qu'en font les historiens de l'époque soviétique remonte aux diatribes de Nikita Hruščev en 1956. Il ajoute que la formule fut vite reprise pour désigner, comme il l'écrit, «la glorification comme quasi-dieu d'un leader politique moderne par le biais de techniques médiatiques et par une adulation populaire excessive ». L'auteur note qu'il suffit d'enlever le terme « leader politique » pour que la définition porte sur n'importe quelle vedette du monde du spectacle.

Par conséquent, ces deux volumes dépassent très largement l'analyse du culte de la personnalité. Cette expression est loin de rendre compte, entre autres, de la tradition et du contexte historique des sociétés qui produisent des guides, d'un panthéon où plusieurs divinités profitent de l'auréole du démiurge, ainsi que d'un système où l'exaltation du grand homme est censée masquer les privations et la terreur. Elle ne fait même pas allusion à la personnalité réelle, aux facultés qui prédisposent un simple mortel à devenir l'objet d'un culte et à la psyché d'un homme d'État qui devient idole. qu'appliquent les acteurs historiques, à partir du moment où le chercheur choisit de ne pas l'expliquer dans son contexte immédiat. Le recours aux stéréotypes discursifs d'une période historique donnée comporte le risque de ne saisir que les représentations collectives d'un temps révolu et d'obscurcir les phénomènes du passé en tant que tels.

20 N'oublions pas cependant que nous assistons à l'ouverture d'un chantier prometteur. Il faut souligner l'aspect pionnier de ces travaux et souhaiter qu'ils soient poursuivis. 